Research Paper: Gerotarget (Focus on Aging)

\title{
Telomere attrition, kidney function, and prevalent chronic kidney disease in the United States
}

\author{
Moshen Mazidi'1,2, Peyman Rezaie ${ }^{1,2}$, Adriac Covic ${ }^{3}$, Jolanta Malyszko4, Jacek Rysz ${ }^{5}$, \\ Andre Pascal Kengne ${ }^{6}$ and Maciej Banach ${ }^{7,8}$ \\ ${ }^{1}$ Key State Laboratory of Molecular Developmental Biology, Institute of Genetics and Developmental Biology, Chinese \\ Academy of Sciences, Beijing, China \\ ${ }^{2}$ Institute of Genetics and Developmental Biology, International College, University of Chinese Academy of Science, Beijing, \\ China \\ ${ }^{3}$ Nephrology Clinic, Parhon University Hospital, Grigore T. Popa University of Medicine and Pharmacy, Iasi, Romania \\ ${ }^{4}$ Department of Nephrology and Hypertension with Dialysis Unit, Medical University of Bialystok, Bialystok, Poland \\ ${ }^{5}$ Department of Nephrology, Hypertension and Family Medicine, Medical University of Lodz, Zeromskiego, Lodz, Poland \\ ${ }^{6}$ Non-Communicable Disease Research Unit, South African Medical Research Council and University of Cape Town, Cape \\ Town, South Africa \\ 7 Department of Hypertension, Chair of Nephrology and Hypertension, Medical University of Lodz, Lodz, Poland \\ ${ }^{8}$ Polish Mother's Memorial Hospital Research Institute (PMMHRI), Lodz, Poland \\ Correspondence to: Mohsen Mazidi, email: Moshen@genetics.ac.cn
}

Keywords: telomere length, kidney function, glomerular filtration rate, national health and nutrition examination survey, albumincreatinine ratio, Gerotarget

Received: February 13, $2017 \quad$ Accepted: May 06, $2017 \quad$ Published: September 08, 2017

Copyright: Mazidi et al. This is an open-access article distributed under the terms of the Creative Commons Attribution License 3.0 (CC BY

3.0), which permits unrestricted use, distribution, and reproduction in any medium, provided the original author and source are credited.

\section{ABSTRACT}

Background: Telomere length is an emerging novel biomarker of biologic age, cardiovascular risk and chronic medical conditions. Few studies have focused on the association between telomere length (TL) and kidney function.

Objective: We investigated the association between TL and kidney function/ prevalent chronic kidney disease (CKD) in US adults.

Methods: The National Health and Nutrition Examination Survey (NHANES) participants with measured data on kidney function and TL from 1999 to 2002 were included. Estimated glomerular filtration rate (eGFR) was based on CKD Epidemiology Collaboration (CKD-EPI) equation. Urinary albumin excretion was assessed using urinary albumin-creatinine ratio (ACR). We used multivariable adjusted linear and logistic regression models, accounting for the survey design and sample weights.

Results: Of the 10568 eligible participants, $48.0 \%(n=5020)$ were men. Their mean age was 44.1 years. eGFR significantly decreased and ACR significantly increased across increasing quarters of TL (all $p<0.001$ ). The association between TL and kidney function remained robust even after adjusting for potential confounding factors, but the association between TL and ACR was only borderline significant ( $\beta$-coefficient= $-0.012, p=0.056$ ).

Conclusion: The association of kidney function with a marker of cellular senescence suggests an underlying mechanism influencing the progression of nephropathy.

\section{INTRODUCTION}

Telomere is a repeat of specific short sequences of nucleotides found at the end of chromosomes. The length of telomere is so considerable, hence telomere shortening has been correlated with various pathological 
disorders and processes such as biological aging, oxidative stress and inflammation [1-6]. The stability of telomere is vital for the conservation of genetic information and maintaining cellular stability [7-9]. Additionally, distortion of telomere can result in cellular abnormality and organ failure $[10,11]$.

There is great interest in studying telomere length in relation with kidney health $[12,13]$, but clinical and epidemiological studies remain scanty. The limited existing evidence suggest that patients with end-stage renal disease (ESRD) may have shorter telomere and accelerated telomere shortening compared with the general population $[14,15]$. Data for subjects with chronic kidney disease (CKD), derived mainly from two studies of severe heart failure patients, also suggest a strong correlation between reduced kidney function and shorter telomere length (TL), even after adjustment for age $[16,17]$.

The mechanism through which creatinine is regulated by the kidney, and the relationship between kidney function and TL are not fully understood, while existing data have been controversial [18]. However, clarifying the association of kidney function with TL is imperative, to confirm if this pathway holds promise for CKD risk evaluation and/or reduction.

In this study we investigated the association of TL, a marker for biological age, with kidney function and prevalent CKD using data from the National Health and Nutrition Examination Survey (NHANES).

\section{RESULTS}

Of the 10568 eligible participants, $48.0 \%(n=5020)$ were men. The mean age was 44.1 years overall, 43.5 years in men and 44.8 years in women $(p=0.063)$. With regard to education $52.5 \%(n=4022)$ of the participants had completed more than high school, $25.6 \%(n=2178)$ had completed high school, while $21.7 \%(n=3248)$ had completed less than high school. White (non-Hispanic) represented $70.4 \%(n=4864)$ of the participants, AfricanAmericans - 10.9\% $(n=2103)$ and Mexican-Americans - 6.9\% ( $n=2690)$. Overall, 20.8\% were current smokers ( $24.8 \%$ of the men and $16.6 \%$ of the women). The mean and standard error of mean (SEM) for the TL in the overall sample was $1.08 \pm 0.015(1.07 \pm 0.014$ in men and $1.08 \pm 0.016$ in women).

The distribution of participants by stage of CKD based on eGFR and ACR levels was the following: CKD stage $1-65.3 \%$, CKD stage $2-26.5 \%$, CKD stage $3-$ $7.1 \%$, CKD stage $4-0.6 \%$, and CKD stage $5-0.4 \%$. Overall, $8.1 \%$ of the participants had eGFR less than $60 \mathrm{ml} / \mathrm{min} / 1.73 \mathrm{~m}^{2}$. Table 1 shows the characteristics of the participants according to their status for CKD. The lipid profile including triglyceride, total cholesterol, high-density lipoprotein cholesterol, and low-density lipoprotein cholesterol, was better in participants without CKD than in those with CKD (all $p<0.001)$.
The association age- and sex-adjusted cardiometabolic factors, kidney function tests across quarters of TL analyzed are summarized in Table 2. Mean body mass index, fat-free mass, fat mass, triglyceride, total cholesterol and C-reactive protein significantly decreased across increasing TL quarters (all $p<0.001$ ), while highdensity lipoprotein cholesterol significantly increased across increasing TL quarters ( $p<0.001$, Table 2$)$. eGFR significantly decreased and ACR significantly increased across increasing quarters of TL (both $p<0.001$ ).

Further univariable and multivariable (age-, sex-, race-, smoking-, fasting blood glucose-, systolic and diastolic blood pressure-, body mass index-, and C-reactive protein) regression analysis were performed to examine the association of TL with kidney function (Table $3)$. Univariable models revealed that TL was negatively associated with urea albumin and ACR (both $p<0.001$ ), and positively associated with serum creatinine and eGFR (both $p<0.001$ ). In multivariable adjusted models, the association remained significant between TL and eGFR, and borderline significant between TL and Urea Albumin $(\beta$-coefficient $=-0.012, p=0.056)$. Logistic regression was used to determine the association between quartile of the TL and chance of CKD, however we have failed to find any significant association between quartile of the TL and odds of CKD neither in crude nor in adjusted (age, sex-, race-, smoking-, fasting blood glucose-, systolic and diastolic blood pressure-, body mass index-, and C-reactive protein) models.

\section{DISCUSSION}

In this large representative sample of adults Americans, eGFR decreased while urinary albumin excretion increased across decreasing TL quarters. These patterns were robust to adjustment for potential confounding factors. Although, these findings did not translate into significant association between TL and prevalent CKD, our study findings suggest that telomere shortening could be an independent predictor of deteriorating kidney function.

In accordance with our findings, recent systematic review proposed that shortening TL might be related with CKD prevalence/occurrence or declining kidney function, however this relation is probably balanced by the cellular telomere reparative process in those surviving longer with CKD, furthermore stated that Short TL was independently related with increased risk of prevalent micro albuminuria in diabetic men with CKD [19]. Furthermore, recent Japanese investigation among persons with increased cardiovascular risk, telomere length indicated a relationship of longer telomere length to better renal function [20].

However, Pykhtina etal stated that no associations have been found between telomere length and inflammatory markers and the levels of glomerular 
Table 1: Antrpometrical and clinical characters of subjects based on CKD status

\begin{tabular}{|l|c|c|c|}
\hline \multicolumn{1}{|c|}{ Variables } & \multicolumn{2}{c|}{ CKD Status } \\
\cline { 2 - 4 } & \multirow{2}{*}{ CKD Positive } & \multirow{2}{*}{ CKD Negative } & \multicolumn{1}{c|}{$p$-value } \\
\hline Body Mass Index (kg/m $\mathbf{m}^{\mathbf{2}}$ & $30.2 \pm 2.32$ & $27.5 \pm 3.52$ & $<0.001$ \\
\hline Fat-free mass (kg) & $55.4 \pm 3.25$ & $54.7 \pm 2.86$ & 0.096 \\
\hline Fat mass (kg) & $29.1 \pm 4.12$ & $25.4 \pm 1.43$ & $<0.001$ \\
\hline Triglyceride (mg/dl) & $174.1 \pm 5.82$ & $149.2 \pm 7.49$ & $<0.001$ \\
\hline Low density lipoprotein cholesterol(mg/dl) & $135.1 \pm 1.46$ & $110.3 \pm 3.56$ & $<0.001$ \\
\hline Total cholesterol(mg/dl) & $210.1 \pm 0.95$ & $179.5 \pm 1.82$ & $<0.001$ \\
\hline High density lipoprotein cholesterol(mg/dl) & $44.6 \pm 0.76$ & $52.9 \pm 0.82$ & $<0.001$ \\
\hline Systolic blood pressure & $132.5 \pm 0.26$ & $119.6 \pm 0.24$ & 0.079 \\
\hline Diastolic blood pressure & $72.8 \pm 1.02$ & $64.1 \pm 0.98$ & $<0.001$ \\
\hline Fasting blood glucose(mg/dl) & $112.3 \pm 2.86$ & $98.6 \pm 2.41$ & $<0.001$ \\
\hline C-reactive protein(mg/dL) & $0.63 \pm 0.01$ & $0.40 \pm 0.02$ & $<0.001$ \\
\hline Log Cystatin C (mg/L) & $0.48 \pm 0.02$ & $0.16 \pm 0.01$ & $<0.001$ \\
\hline Urea Albumin (ug/mL) & $296.5 \pm 4.12$ & $23.9 \pm 2.82$ & $<0.001$ \\
\hline Glomerular filtration rate (mL/min) & $40.3 \pm 0.69$ & $112.3 \pm 0.41$ & $<0.001$ \\
\hline Log Albumin-Creatinine Ratio (mg/dl) & $2.0 \pm 0.01$ & $1.8 \pm 0.01$ & $<0.001$ \\
\hline CKD: chronic kidney disease & & \\
\hline
\end{tabular}

Table 2: Age-, sex-adjusted mean of kidney function tests level across quartiles of telomere length

\begin{tabular}{|c|c|c|c|c|c|}
\hline \multirow{2}{*}{ Variables } & \multicolumn{4}{|c|}{ Quarters of telomere length } & $\mathbf{P}-$ \\
\hline & Q1 & $\mathbf{Q 2}$ & Q3 & Q4 & \multirow{2}{*}{ trend $\dagger$} \\
\hline TL, Mean \pm SEM (T/S ratio) & $0.73 \pm 0.02$ & $0.92 \pm 0.01$ & $1.08 \pm 0.01$ & $1.38 \pm 0.06$ & \\
\hline Body Mass Index $\left(\mathrm{kg} / \mathrm{m}^{2}\right)$ & $28.5 \pm 0.18$ & $28.1 \pm 0.19$ & $27.8 \pm 0.42$ & $26.4 \pm 0.21$ & $<0.001$ \\
\hline Fat-free mass (kg) & $54.8 \pm 0.94$ & $54.4 \pm 0.96$ & $53.4 \pm 0.74$ & $51.0 \pm 0.68$ & $<0.001$ \\
\hline Fat mass (kg) & $26.9 \pm 0.89$ & $25.8 \pm 0.32$ & $24.9 \pm 0.76$ & $24.1 \pm 0.96$ & $<0.001$ \\
\hline Triglyceride (mg/dl) & $150.7 \pm 5.7$ & $142.1 \pm 6.2$ & $140.1 \pm 5.9$ & $133.0 \pm 5.1$ & $<0.001$ \\
\hline Low density lipoprotein cholesterol(mg/dl) & $123.0 \pm 1.95$ & $124.1 \pm 1.49$ & $122.0 \pm 1.29$ & $124.1 \pm 1.46$ & 0.615 \\
\hline Total cholesterol(mg/dl) & $203.1 \pm 1.9$ & $199.6 \pm 1.07$ & $189.3 \pm 1.92$ & $180.7 \pm 1.46$ & $<0.001$ \\
\hline High density lipoprotein cholesterol(mg/dl) & $49.3 \pm 0.62$ & $51.1 \pm 0.49$ & $52.1 \pm 0.73$ & $54.0 \pm 0.82$ & $<0.001$ \\
\hline Systolic blood pressure & $122.0 \pm 0.68$ & $121.3 \pm 0.66$ & $122.5 \pm 0.39$ & $122.8 \pm 0.54$ & 0.169 \\
\hline Diastolic blood pressure & $72.06 \pm 0.54$ & $73.2 \pm 0.40$ & $73.8 \pm 0.62$ & $72.4 \pm 0.31$ & 0.069 \\
\hline Fasting blood glucose(mg/dl) & $101.0 \pm 1.2$ & $101.3 \pm 1.05$ & $102.4 \pm 1.82$ & $101.9 \pm 2.31$ & 0.905 \\
\hline C-reactive protein(mg/dL) & $0.53 \pm 0.02$ & $0.46 \pm 0.01$ & $0.40 \pm 0.01$ & $0.32 \pm 0.03$ & $<0.001$ \\
\hline Log Cystatin C (mg/L) & $0.90 \pm 0.01$ & $0.083 \pm 0.01$ & $0.80 \pm 0.02$ & $0.74 \pm 0.02$ & $<0.001$ \\
\hline Urea Albumin (ug/mL) & $44.9 \pm 5.36$ & $39.5 \pm 8.68$ & $32.8 \pm 7.36$ & $27.4 \pm 4.43$ & $<0.001$ \\
\hline Glomerular filtration rate $(\mathrm{mL} / \mathrm{min})$ & $96.5 \pm 0.92$ & $99.9 \pm 0.74$ & $101.4 \pm 0.64$ & $106.2 \pm 0.69$ & $<0.001$ \\
\hline Log Albumin-Creatinine Ratio (mg/dl) & $3.16 \pm 0.03$ & $2.96 \pm 0.03$ & $2.43 \pm 0.03$ & $1.95 \pm 0.02$ & $<0.001$ \\
\hline
\end{tabular}

${ }^{a} P$-values for linear trend across quarters of telomere length. Variables were compared across quartiles of telomere length using analysis of variance (ANOVA) test .CKD: chronic kidney disease.

filtration rate, urea and creatinine except for albuminuria which is associated with telomere length [21].

In line with our study, previous reports have suggested that TL is shorter in patients with end-stage renal disease patients on dialysis compared with the general population. For example, in a study of 15 patients on haemodialysis and 15 age-matched controls, the authors found an accelerated telomere shortening in patients on dialysis [22]. Another study in 18 diabetic patients on dialysis and 20 controls found an inverse correlation between TL and length of time in dialysis [14]. A study of 42 haemodialysis patients found reduced telomerase activity compared with non-haemodialysis patients [15].

Interestingly, recent investigation conducetd by Karin Luttropp concluded that Telomere attrition after 12 months was significantly greater in patients with 
Table 3: Univariate and multivariate (age-, sex-, race-, smoking-, fasting blood glucose-, systolic and diastolic blood pressure-, body mass index-, C-reactive protein, diabetes and hypertension) of association between kidney function tests level and telomere length

\begin{tabular}{|l|c|c|c|c|}
\hline \multirow{2}{*}{ Variables } & \multicolumn{2}{c|}{ Univariate } & \multicolumn{2}{c|}{ Multivariate } \\
\cline { 2 - 5 } & B-coefficient & $\boldsymbol{p}$-value & B- coefficient & $\boldsymbol{p}$-value \\
\hline Urea Albumin (ug/mL) & -0.094 & $<0.001$ & -0.09 & 0.069 \\
\hline Log Cystatin C (mg/L) & -0.264 & $<0.001$ & 0.141 & $<0.001$ \\
\hline Glomerular filtration rate (mL/min) & 0.302 & $<0.001$ & 0.195 & $<0.001$ \\
\hline Log Albumin-Creatinine Ratio (mg/dl) & -0.142 & $<0.001$ & -0.131 & 0.025 \\
\hline
\end{tabular}

renal replacement therapy compared to dialysis patients in addition non-CKD patients had meaningfully longer telomeres than CKD patients [23].

regarding to the evidence from pre-ESRD and CKD patients, earlier studies in patients with heart failure and normal range kidney function have reported a strong correlation between shortening TL and declining kidney function, even after adjustment for age $[16,17]$. In this regard, Pim et al, have evaluated association of TL with renal function in 610 patients with heart failure (aged 40 to 80 years), and found that age-and sex-adjusted TL decreased steadily across decreasing quarters of eGFR [16]. Another study by Wong et al, explored the association between TL and renal function in patients with chronic heart failure $(n=866$, median age was 74$)$ [17]. They reported that TL was associated with renal function, even after adjustment for age, gender, age at chronic heart failure onset, and severity of chronic heart failure [17]. In contrast, the Heart and Soul study which is longitudinal study of patients with stable coronary heart disease, found that kidney function was not independently associated with shortened TL or telomere shortening over 5 years [24]. However their findings should be considered with caution, considering the focus on relatively old predominantly subjects (mean age was 66.7 ), with stable coronary heart disease [24].

This study has several strengths. It is one of the largest studies of the association of TL with kidney impairment. Kidney impairment was assessed by both eGFR and proteinuria. The selection of participants was based on random sampling of the general population and therefore the results can be extrapolated to the general population. As the data collection was performed on all days of the week throughout the year in NHANES, the potential for selection bias is very low $[25,26]$. The findings from our study should be considered in the context of some limitations. The cross-sectional nature does not allow inference about causality. We did not have available any repeated measure of TL with quantitative polymerase chain reaction in the same subjects after several follow-up years to elucidate temporality of these findings.

This study has important clinical and public health implications. Understanding the interplay between TL and kidney function is a necessary and important step toward any application of the resulting knowledge for public health policy and action. Moreover we knew that CKD may increase the risk of CVD as leading cause of the death which may could be explain by role of the TL [27-29]. Our study provides a comprehensive snapshot of the relationship of kidney function with TL at the national level in the US.

In conclusion, our findings provide further evidence on the association between TL and kidney function. The association of kidney function with a marker of cellular senescence suggests an underlying mechanism influencing the progression of nephropathy.

\section{MATERIALS AND METHODS}

\section{Population}

The NHANES are ongoing repeated cross-sectional surveys conducted by the US National Center for Health Statistics (NCHS). The NCHS Research Ethics Review Board approved the NHANES protocol and consent was obtained from all participants [30, 31]. Data collection on demographic, diets, and behaviours occurred through questionnaires administered during home visits, while anthropometrics and biomarkers data were collected by trained staff using mobile examination units $[30,32]$. The interview consisted of questions on socio-demographic characteristics (age, gender, education, race/Hispanic origin, and health insurance) and questions on previously diagnosed medical conditions. More detailed information on the NHANES protocol is available elsewhere [30, 33, 34]. This study was based on analysis of data from the 1999-2002 NHANES cycles. Analyses were restricted to participants aged 18 years and older. Fasting blood glucose (FBG), total cholesterol (TC), low density lipoprotein cholesterol (LDL-C), high density lipoprotein cholesterol (HDL-C), triglycerides (TG) levels and telomere length were assayed using methods described in the NHANES Laboratory/Medical Technologists Procedures Manual [30, 35, 36]. Complete laboratory procedures for collection, storage, calibration and quality control of blood samples for determination of hsCRP and other inflammatory markers are available elsewhere. 
[37] Creatinine was measured by the Jaffe reaction and standardized by methods described previously [38]. A random urine specimen was collected from participants, and urinary creatinine was measured by the Jaffe rate reaction, urinary albumin was measured by solidphase fluorescent immunoassay [39]. Albuminuria was measured by urinary albumin-creatinine ratio (ACR) [39]. Glomerular filtration rate [eGFR, $\left(\mathrm{ml} / \mathrm{min} / 1.73 \mathrm{~m}^{2}\right)$ ] was estimated using the CKD Epidemiology Collaboration (CKD-EPI) equation. CKD was defined as eGFR less than $60\left(\mathrm{ml} / \mathrm{min} / 1.73 \mathrm{~m}^{2}\right)$ [39].

\section{Telomere measurements}

Aliquots of purified DNA, isolated from whole blood using the Puregene (D-50 K) kit protocol (Gentra Systems, Inc., Minneapolis, MN, USA), were obtained from participants. TL assay was performed using the quantitative polymerase chain reaction method to measure TL relative to standard reference DNA (also known as the telomere-to-single copy gene (T/S) ratio) $[35,40]$. Each sample was assayed 3 times on 3 different days. The samples were assayed on duplicate wells, resulting in 6 data points. Control DNA values were used to normalize between-run variability [40, 41]. Runs with more than 4 control DNA values falling outside 2.5 standard deviations from the mean for all assay runs were excluded from further analysis ( $6 \%$ of runs). For each sample, any potential outliers were identified and excluded from the calculations ( $2 \%$ of samples). The inter-assay coefficient of variation was $6.5 \%$. The Centers for Disease Control (CDC) conducted a quality control review before linking the TL data to the NHANES data files.

\section{Statistical analysis}

We conducted the analyses according to the CDC guidelines for analysis of complex NHANES data, accounting for the masked variance and using the proposed weighting methodology [42]. To investigate the association between TL and kidney function, univariable and multivariable (age-, sex-, race-, smoking-, fasting blood glucose-, systolic and diastolic blood pressure-, body mass index-, C-reactive protein, diabetes and hypertension) regressions were applied. Adjusted (age- and sex-) mean of cardiometabolic factors and kidney function were compared across quarters of TL using the analysis of co-variance (ANCOVA) with Bonferroni correction. Variables were compared by using analysis of variance (ANOVA) and Chi-square tests. All tests were two sided, and $p<0.05$ used to characterise statistically significant results. Data were analysed using SPSS $^{\circledR}$ complex sample module version 22.0 (IBM Corp, Armonk, NY, USA).

\section{ACKNOWLEDGMENTS}

MM was supported by The World Academy of Sciences studentship of the Chinese Academy of Sciences.

\section{CONFLICTS OF INTEREST}

None.

\section{REFERENCES}

1. Blackburn EH. Structure and function of telomeres. Nature. 1991; 350:569-73.

2. Zakian VA. Telomeres: beginning to understand the end. Science. 1995; 270:1601-07.

3. Maeda T, Guan JZ, Higuchi Y, Oyama J, Makino N. Aging-related alterations of subtelomeric methylation in sarcoidosis patients. J Gerontol A Biol Sci Med Sci. 2009; 64:752-60.

4. Iwama H, Ohyashiki K, Ohyashiki JH, Hayashi S, Yahata N, Ando K, Toyama K, Hoshika A, Takasaki M, Mori M, Shay JW. Telomeric length and telomerase activity vary with age in peripheral blood cells obtained from normal individuals. Hum Genet. 1998; 102:397-402.

5. Guan JZ, Maeda T, Sugano M, Oyama J, Higuchi Y, Makino N. Change in the telomere length distribution with age in the Japanese population. Mol Cell Biochem. 2007; 304:353-60.

6. Mazidi M, Kengne AP, Banach M. Mineral and vitamins consumption is associated with longer telomeres among US adults. Pol Arch Intern Med. 2017; 127:87-90. https://doi. org/10.20452/pamw.3927.

7. Blackburn EH. Switching and signaling at the telomere. Cell. 2001; 106:661-73.

8. Pooley KA, Sandhu MS, Tyrer J, Shah M, Driver KE, Luben RN, Bingham SA, Ponder BA, Pharoah PD, Khaw KT, Easton DF, Dunning AM. Telomere length in prospective and retrospective cancer case-control studies. Cancer Res. 2010; 70:3170-76.

9. Artandi SE. Telomeres, telomerase, and human disease. N Engl J Med. 2006; 355:1195-97.

10. Yehezkel S, Segev Y, Viegas-Péquignot E, Skorecki K, Selig S. Hypomethylation of subtelomeric regions in ICF syndrome is associated with abnormally short telomeres and enhanced transcription from telomeric regions. Hum Mol Genet. 2008; 17:2776-89.

11. Mazidi M, Kengne AP, Sahebkar A, Banach M. Telomere Length Is Associated With Cardiometabolic Factors in US Adults. Angiology. 2017; 3319717712860:3319717712860.

12. Song J, Czerniak S, Wang T, Ying W, Carlone DL, Breault DT, Humphreys BD. Characterization and fate of telomerase-expressing epithelia during kidney repair. J Am Soc Nephrol. 2011; 22:2256-65.

13. Westhoff JH, Schildhorn C, Jacobi C, Hömme M, Hartner 
A, Braun H, Kryzer C, Wang C, von Zglinicki T, Kränzlin B, Gretz N, Melk A. Telomere shortening reduces regenerative capacity after acute kidney injury. J Am Soc Nephrol. 2010; 21:327-36.

14. Boxall MC, Goodship TH, Brown AL, Ward MC, von Zglinicki T. Telomere shortening and haemodialysis. Blood Purif. 2006; 24:185-89.

15. Tsirpanlis G, Chatzipanagiotou S, Boufidou F, Kordinas V, Alevyzaki F, Zoga M, Kyritsis I, Stamatelou K, Triantafyllis G, Nicolaou C. Telomerase activity is decreased in peripheral blood mononuclear cells of hemodialysis patients. Am J Nephrol. 2006; 26:91-96.

16. van der Harst P, Wong LS, de Boer RA, Brouilette SW, van der Steege G, Voors AA, Hall AS, Samani NJ, Wikstrand J, van Gilst WH, van Veldhuisen DJ, and MERIT-HF Study Group. Possible association between telomere length and renal dysfunction in patients with chronic heart failure. Am J Cardiol. 2008; 102:207-10.

17. Wong LS, van der Harst P, de Boer RA, Codd V, Huzen J, Samani NJ, Hillege HL, Voors AA, van Gilst WH, Jaarsma $\mathrm{T}$, van Veldhuisen DJ. Renal dysfunction is associated with shorter telomere length in heart failure. Clin Res Cardiol. 2009; 98:629-34.

18. Feig DI, Kang DH, Johnson RJ. Uric acid and cardiovascular risk. N Engl J Med. 2008; 359:1811-21.

19. Ameh OI, Okpechi IG, Dandara C, Kengne AP. Association Between Telomere Length, Chronic Kidney Disease, and Renal Traits: A Systematic Review. OMICS. 2017; 21:14355.

20. Eguchi K, Honig LS, Lee JH, Hoshide S, Kario K. Short telomere length is associated with renal impairment in Japanese subjects with cardiovascular risk. PLoS One. 2017; 12:e176138.

21. Pykhtina VS, Strazhesko ID, Tkacheva ON, Akasheva DU, Dudinskaya EN, Vygodin VA, Plokhova EV, Kruglikova AS, Boitsov SA. [Association of renal function, telomere length and markers of chronic inflammation for patients without chronic kidney and cardiovascular diseases]. Adv Gerontol. 2016; 29:79-85.

22. Ramírez R, Carracedo J, Soriano S, Jiménez R, MartínMalo A, Rodríguez M, Blasco M, Aljama P. Stress-induced premature senescence in mononuclear cells from patients on long-term hemodialysis. Am J Kidney Dis. 2005; 45:35359.

23. Luttropp K, Nordfors L, McGuinness D, Wennberg L, Curley H, Quasim T, Genberg H, Sandberg J, Sönnerborg I, Schalling M, Qureshi AR, Bárány P, Shiels PG, Stenvinkel P. Increased Telomere Attrition After Renal Transplantation-Impact of Antimetabolite Therapy. Transplant Direct. 2016; 2:e116.

24. Bansal N, Whooley MA, Regan M, McCulloch CE, Ix JH, Epel E, Blackburn E, Lin J, Hsu CY. Association between kidney function and telomere length: the heart and soul study. Am J Nephrol. 2012; 36:405-11. https://doi. org/10.1159/000343495.
25. Tooze JA, Midthune D, Dodd KW, Freedman LS, KrebsSmith SM, Subar AF, Guenther PM, Carroll RJ, Kipnis V. A new statistical method for estimating the usual intake of episodically consumed foods with application to their distribution. J Am Diet Assoc. 2006; 106:1575-87.

26. Guenther PM, Ding EL, Rimm EB. Alcoholic beverage consumption by adults compared to dietary guidelines: results of the National Health and Nutrition Examination Survey, 2009-2010. J Acad Nutr Diet. 2013; 113:546-50.

27. Gluba-Brzózka A, Michalska-Kasiczak M, Franczyk B, Nocuń M, Toth P, Banach M, Rysz J. Markers of increased atherosclerotic risk in patients with chronic kidney disease: a preliminary study. Lipids Health Dis. 2016; 15:22.

28. Franczyk-Skóra B, Gluba-Brzózka A, Wranicz JK, Banach M, Olszewski R, Rysz J. Sudden cardiac death in CKD patients. Int Urol Nephrol. 2015; 47:971-82.

29. Colantonio LD, Baber U, Banach M, Tanner RM, Warnock DG, Gutiérrez OM, Safford MM, Wanner C, Howard G, Muntner P. Contrasting Cholesterol Management Guidelines for Adults with CKD. J Am Soc Nephrol. 2015; 26:1173-80.

30. Mazidi M, Michos ED, Banach M. The association of telomere length and serum 25-hydroxyvitamin D levels in US adults: the National Health and Nutrition Examination Survey. Arch Med Sci. 2017; 13:61-65.

31. Mazidi M, Kengne AP, Mikhailidis DP, Cicero AF, Banach M. Effects of selected dietary constituents on highsensitivity C-reactive protein levels in U.S. adults. Ann Med. 2017:1-6.

32. Mazidi M, Pennathur S, Afshinnia F. Link of dietary patterns with metabolic syndrome: analysis of the National Health and Nutrition Examination Survey. Nutr Diabetes. 2017; 7:e255.

33. Klein-Platat C, Drai J, Oujaa M, Schlienger JL, Simon C. Plasma fatty acid composition is associated with the metabolic syndrome and low-grade inflammation in overweight adolescents. Am J Clin Nutr. 2005; 82:1178-84.

34. Mazidi M, Gao HK, Vatanparast H, Kengne AP. Impact of the dietary fatty acid intake on C-reactive protein levels in US adults. Medicine (Baltimore). 2017; 96:e5736.

35 Needham BL, Adler N, Gregorich S, Rehkopf D, Lin J, Blackburn EH, Epel ES. Socioeconomic status, health behavior, and leukocyte telomere length in the National Health and Nutrition Examination Survey, 1999-2002. Soc Sci Med. 2013; 85:1-8.

36. Remer $T$. Influence of nutrition on acid-base balancemetabolic aspects. Eur J Nutr. 2001; 40:214-20.

37. http://www.cdc.gov/NCHS/data/nhanes/nhanes_09_10/ CRP_F_met.pdf. [accessed 19.08.13].

38. Selvin E, Manzi J, Stevens LA, Van Lente F, Lacher DA, Levey AS, Coresh J. Calibration of serum creatinine in the National Health and Nutrition Examination Surveys (NHANES) 1988-1994, 1999-2004. Am J Kidney Dis. 2007; 50:918-26. 
39. Chavers BM, Simonson J, Michael AF. A solid phase fluorescent immunoassay for the measurement of human urinary albumin. Kidney Int. 1984; 25:576-78.

40. Cawthon RM. Telomere length measurement by a novel monochrome multiplex quantitative PCR method. Nucleic Acids Res. 2009; 37:e21.

41. Kimura M, Stone RC, Hunt SC, Skurnick J, Lu X, Cao X,
Harley CB, Aviv A. Measurement of telomere length by the Southern blot analysis of terminal restriction fragment lengths. Nat Protoc. 2010; 5:1596-607.

42. Mazidi M, Gao HK, Shivappa N, Wirth MD, Hebert JR, Kengne AP. The relationship of plasma Trans fatty acids with dietary inflammatory index among US adults. Lipids Health Dis. 2017; 16:147. 